\begin{tabular}{|c|l|}
\hline Title & Crystal structure of cytochrome P450 MoxA from Nonomuraea recticatena (CY P105) \\
\hline Author(s) & Y asutake, Y oshiaki; Imoto, Noriko; Fujii, Y oshikazu; Fujii, Tadashi; A risawa, A kira; Tamura, Tomohiro \\
\hline Citation & $\begin{array}{l}\text { Biochemical and Biophysical Research Communications, 361(4), 876-882 } \\
\text { https://doi.org/10.1016/.bbrc.2007.07.062 }\end{array}$ \\
\hline Issue Date & $2007-10$-05 \\
\hline Doc URL & http://hdl.handle.net/2115/30116 \\
\hline Type & article (author version) \\
\hline File Information & BBRC361-4.pdf \\
\hline
\end{tabular}

Instructions for use 


\title{
Crystal structure of cytochrome P450 MoxA from Nonomuraea recticatena (CYP105)
}

\author{
Yoshiaki Yasutake ${ }^{a}$, Noriko Imoto ${ }^{b}$, Yoshikazu Fujii ${ }^{c}$, Tadashi Fujii ${ }^{c}$, Akira \\ Arisawa $^{c}$, and Tomohiro Tamura ${ }^{\mathrm{a}, \mathrm{b}, *}$ \\ ${ }^{a}$ Research Institute of Genome-based Biofactory, National Institute of Advanced \\ Industrial Science and Technology (AIST), 2-17-2-1 Tsukisamu-Higashi, Toyohira-ku, \\ Sapporo 062-8517, Japan \\ ${ }^{b}$ Laboratory of Molecular Environmental Microbiology, Graduate School of Agriculture, \\ Hokkaido University, Kita-9, Nishi-9, Kita-ku, Sapporo 060-8589, Japan \\ ${ }^{c}$ Bioresource Laboratories, Mercian Corporation, 1808 Nakaizumi, Iwata, Shizuoka \\ 438-0078, Japan
}

*Corresponding author: T. Tamura; Tel, 81-11-857-8938; Fax, 81-11-857-8980;

E-mail, t-tamura@aist.go.jp

\begin{abstract}
Abbreviations: AU, asymmetric unit; L-BE, Luciferin 6'-benzyl ether; L-CEE, Luciferin 6'-chloroethyl ether; L-H, 6'-deoxyluciferin; L-H-EGE, Ethylene glycol ester of L-H; L-ME, Luciferin 6'-methyl ether; L-ME-EGE, Ethylene glycol ester of L-ME; MES, 2-( $N$-morpholino) ethanesulfonic acid; PAGE, polyacrylamide gel electrophoresis; rmsd, root-mean-square deviations; SDS, sodium dodecyl sulfate; Tris, 2-amino-2-(hydroxymethyl)-1,3-propanediol.
\end{abstract}




\begin{abstract}
Cytochrome P450 MoxA (P450moxA) from a rare actinomycete Nonomuraea recticatena belongs to the CYP105 family and exhibits remarkably broad substrate specificity. Here, we demonstrate that $\mathbf{P 4 5 0 m o x A}$ acts on several luciferin derivatives, which were originally identified as substrates of the human microsomal P450s. We also describe the crystal structure of P450moxA in substrate-free form. Structural comparison with various bacterial and human microsomal P450s reveals that the $\mathbf{P 4 5 0 m o x A}$ structure is most closely related to that of the fungal nitric oxide reductase P450nor (CYP55A1). Final refined model of $\mathrm{P450moxA}$ comprises almost all the residues, including the "BC-loop" and "FG-loop" regions pivotal for substrate recognition, and the current structure thus defines a well-ordered substrate-binding pocket. Clear electron density map reveals that the MES molecule is bound to the substrate-binding site, and the sixth coordination position of the heme iron is not occupied by a water molecule, probably due to the presence of MES molecule in the vicinity of the heme. The unexpected binding of the MES molecule might reflect the ability of P450moxA to accommodate a broad range of structurally diverse compounds.
\end{abstract}

Key words: Crystal structure; CYP105; Cytochrome P450; MoxA; Nonomuraea recticatena 


\section{Introduction}

Cytochrome P450 monooxygenases (P450s) are a superfamily of heme-containing enzymes that catalyze a wide variety of oxidative reactions, i.e., biosynthesis of steroid hormones, lipids, and complex antibiotics such as polyketides, detoxification of xenobiotics, drug metabolism, and bioconversion of recalcitrant molecules to utilize them as carbon sources [1]. Catalytic reactions include hydroxylation, epoxidation, phenol coupling, heteroatom oxidation, dehalogenation, and carbon-carbon bond cleavage via successive hydroxylations [2]. Functional studies of P450s have a major impact on human physiology; the catalytic abilities of P450s are highly attractive in that they involve a high regio- and stereo-selectivity, thus introducing the possibility of numerous biotechnological applications such as industrial production of valuable chemicals by bioconversion. To identify P450s with useful catalytic activities, we had previously developed a novel P450 expression system by using Escherichia coli that harbors a plasmid carrying both the P450 gene and feasible redox partner genes [3]. To date, we have collected 213 bacterial P450 genes and constructed an expression library for exploring useful P450s that facilitate the conversion to industrially valuable compounds.

P450moxA from the rare actinomycete Nonomuraea recticatena IFO 14525 was first identified as an enzyme that exhinited ML236-B (compactin) hydroxylase activity. A sequence similarity search indicated that P450moxA belongs to the CYP105 family [4]. Recently, several CYP105 P450s have been characterized. P450sca-2 (CYP105A3) from Streptomyces carbophilus also possesses hydroxylation activity to ML236-B for its detoxification [5]. P450SU-1 (CYP105A1) from Streptomyces griseolus is known to metabolize sulfonylurea herbicides [6] and also convert vitamin $\mathrm{D}_{3}$ to 25-hydroxyvitamin $\mathrm{D}_{3}$ [7]. P450soy (CYP105D1) from Streptomyces griseus was found to be deeply involved in the degradation of a diverse array of complex agrochemicals and environmental pollutants $[8,9]$. These observations clearly indicate that the CYP105 enzymes play a crucial role in xenobiotic degradation as well as antibiotic biosynthesis. Interestingly, tests for acceptable substrates have revealed that P450moxA also acts on a wide range of hydrophobic compounds such as testosterone [4] and oleanolic acid [10]. The remarkably broad substrate specificity of the CYP105 enzymes is reminiscent of the mammalian liver microsomal P450s, e.g., CYP2D6 and CYP3A4, which also exhibit broad substrate specificity for metabolizing a vast array of 
xenobiotics (drugs) [11]. Most actinomycetes possess a relatively large number of P450s, including the CYP105s [12], suggesting that they are important for survival in soil.

In this study, we demonstrate that P450moxA possesses the ability to act on certain luciferin derivatives, which were originally identified as substrates for the human liver microsomal P450s (CYP1A1, CYP1A2, CYP1B1, CYP2C8, CYP3A4, CYP3A7, and CYP2D6). Further, the crystal structure of P450moxA has been determined to a resolution of $2.8 \AA$ in the substrate-free form. This is the first report of the X-ray structure of CYP105, and it may serve as a model for further structural study and molecular dynamics simulation analysis of CYP105 and related P450s. 


\section{Materials and methods}

\section{Plasmid construction and expression in Rhodococcus erythropolis.}

Identification and cloning of the P450moxA gene (moxA) have been described previously [4]. The moxA gene and its downstream ferredoxin gene $(\operatorname{mox} B)$ were inserted into the pTipQT vector $[13,14]$ together with the $R$. erythropolis NI86/21 ferredoxin and ferredoxin reductase genes thc $C$ and $t h c D$ [15]. A ribosome-binding site was added immediately upstream of thc $C D$ in order to facilitate translation. The resulting plasmid pTipQT-MoxAB-ThcCD was introduced into the $R$. erythropolis JCM2301 cells by electropolation. For details, see Supplementary methods.

P450 assay by the luminescent method. The $\mathrm{P} 450$ assay was performed using the P450-Glo ${ }^{\mathrm{TM}}$ assay system (Promega). Six luciferin derivatives, namely, luciferin 6'-chloroethyl ether (L-CEE), luciferin 6'-methyl ether (L-ME), 6'-deoxyluciferin (L-H), luciferin 6'-benzyl ether (L-BE), ethylene glycol ester of L-ME (L-ME-EGE) and ethylene glycol ester of L-H (L-H-EGE), were tested as luminogenic substrates for the assay. The assays were performed according to the manufacturer's instructions. A reaction mixture prepared by mixing $35 \mu \mathrm{l}$ of cell-free extracts (containing $300 \mu \mathrm{g}$ of total proteins), $5 \mu \mathrm{l}$ of $40 \mathrm{mM} \mathrm{NADH}$, and $5 \mu \mathrm{l}$ of each substrate $(20-100 \mu \mathrm{M})$ was incubated for $6 \mathrm{hr}$ at $30^{\circ} \mathrm{C}$. Luminescence was measured using a luminometer (Centro LB 960; Berthold Technologies) after adding $40 \mu$ of luciferin detection reagent to each reaction mixture.

X-ray structure analysis. Recombinant $\mathrm{P} 450$ moxA with a His-tag at its C-terminus was overexpressed using E. coli, and the enzyme was purified by Ni-affinity chromatography. The initial crystallization screening was performed by the vapor-diffusion technique at $20^{\circ} \mathrm{C}$, using the commercially available sparse-matrix screens. Subsequent optimization of the initial conditions was performed with varying $\mathrm{pH}$, precipitant concentrations, and the use of various additives. The rod-shaped single crystals $(0.3 \mathrm{~mm} \times 0.05 \mathrm{~mm} \times 0.05 \mathrm{~mm})$ were obtained with a reservoir consisting of 0.1 M 2-( $N$-morpholino) ethanesulfonic acid (MES) (pH 6.5), 8\% ethyleneglycol, $3.5-6 \%$ polyethylene glycol (PEG) 8000 , and $15-20 \%$ glycerol. The X-ray diffraction data were measured at beamline BL6A of the Photon Factory (PF; Tsukuba, Japan). All the data were processed with the program MOSFLM [16] and SCALA [17]. The 
structure of P450moxA was solved by the molecular replacement (MR) method using the program AMoRe [18], applying the truncated structure of the P450eryF (CYP107A1; PDB code, 1jin) [19] as a search model. The program XtalView/Xfit [20] was employed for model building and fitting. Refinement was performed using the program CNS ver. 1.1 [21]. The data collection and refinement statistics are provided in Table 1. For details, see Supplementary methods. The atomic coordinates of P450moxA were deposited in Protein Data Bank under accession code 2z36. 


\section{Results and discussion}

\section{P450 assay with 6 luminogenic substrates}

Successful coexpression of P450moxA and the feasible redox partner ThcCD in R. erythropolis was induced by thiostrepton (Fig. 1(A)). The cell-free extracts were tested for P450-Glo ${ }^{\mathrm{TM}}$ assay system (Promega) [22] by using the 6 available luminogenic substrates. The results clearly indicated that P450moxA possessed activity for the 4 substrates L-CEE, L-ME, L-BE, and L-ME-EGE, which were originally identified as substrates for the human microsomal P450s, namely, CYP1A1, CYP1A2, CYP1B1, CYP2C8, CYP2D6, CYP3A4, and CYP3A7. In contrast, no activity was detected for L-H and L-H-EGE (Fig. 1(B)). The catalytic reactions in this assay were not simple hydroxyl group insertions to the substrates as observed for many bacterial P450s but were the following elimination reactions: dechloroethylation of L-CEE, demethylation of L-ME and L-ME-EGE, and debenzylation of L-BE. Such cleavage activity was not very common in both prokaryotic and eukaryotic P450s [23] and have not been reported in the case of the CYP105 enzymes thus far. The debenzylation activity of L-BE is particularly interesting because the enzyme eliminated the relatively bulky benzyl group. This implied that a large hydrophobic cavity is created in the proximity of the heme, which might enable capture of a wide range of compounds at the substrate binding site.

\section{Crystal structure of P450moxA}

The crystal structure of P450moxA was determined using the MR method and refined at a resolution of $2.8 \AA$ with reasonable stereochemical quality. The crystallographic $R$-factor $\left(R_{\text {work }}\right)$ and $R$-free factor $\left(R_{\text {free }}\right)$ converged to $23.0 \%$ and $27.4 \%$, respectively. The AU contains 2 P450moxA molecules (chains A and B) with a solvent content, $62.2 \%$. No conformational differences were observed between these 2 chains in the $\mathrm{AU}$; the root-mean-square deviation (rmsd) for the main chain atoms (residues 10-412) was $0.27 \AA$. The overall structure of the P450moxA exhibits a typical P450 fold, as illustrated in Fig. 2(A). The molecule is described to be triangular in shape with $55-\AA$ long sides and of $30-\AA$ thick. The structure comprises $14 \alpha$-helices $\left(\alpha \mathrm{A}-\alpha \mathrm{L}, \alpha \mathrm{B}^{\prime}\right.$ and $\left.\alpha \mathrm{K}^{\prime}\right)$ and $6 \beta$-strands $(\beta 1-\beta 6)$, and it is roughly divided into $\alpha$-rich and $\beta$-rich regions. The most distinguishing feature of the P450 fold is an I-helix with a length of almost 50 $\AA$ that runs across the entire molecule. The heme is embedded in the proximal I-helix 
and distal L-helix, and the Cys356 derived from the N-terminal of the L-helix serves as the fifth axial thiolate ligand to the heme iron. The molecules have a well-defined electron density map except for $\mathrm{N}$-terminal 8 residues in chain $\mathrm{A}, 7$ residues in chain $\mathrm{B}$, and for C-terminal 1 residue in both the chains; the models for these could not be established. Electron density for 5 of the 6 histidine residues (residues 408-412) at the C-terminal His-tag sequence was clearly observed due to the presence of the metal ion detected in the $m F_{\mathrm{O}}-D F \mathrm{c}$ map with approximately $11.0 \sigma$ level. The metal is coordinated in a tetrahedral geometry by side-chains of His410, His412, His132' and Asp136' (prime sign indicates the residues belonging to other molecules in the AU). A database search for the metal coordination groups [24] suggested that the bound metal was most probably Fe. The crystallization mother liquor contained no heavy metals such as $\mathrm{Zn}, \mathrm{Fe}$, or $\mathrm{Cu}$, and it is likely that $\mathrm{Fe}$ originated as a result of the degradation of the heme in the crystallization droplets during prolonged storage. Additionally, the MES molecules were found to bind to the substrate-binding site (see the substrate-binding site).

\section{Structural comparison with other known P450s}

The phylogenetic tree reveals that CYP105 is closely related to the CYP55 and CYP107. This evolutionary relationship is consistent with the results of the 3D structure superimpositions, as shown in Fig. 2 (B). The pairwise structure comparison utilizing the DALI server [25] demonstrates that P450moxA is the most structurally homologous to the fungal nitric oxide reductase P450nor (CYP55A1 [26-28]) (PDB code, 1ROM; Z score, 52.6; rmsd, $1.7 \AA$ for $393 \mathrm{C} \alpha$ atoms; sequence identity for fit regions, $37 \%$ ). Here, the notable finding was that despite the functional differences between P450moxA and P450nor, the main-chain structure, including the BC-loop and FG-helices, was very similar to each other. P450nor accommodates NADH at the substrate-binding pocket that enables direct hydride transfer to the NO-bound heme, and the 2 arginine residues (Arg64 at the F-helix and Arg174 at the BC-loop) are considered to be important for the binding of the pyrophosphate moiety of the bound NADH [28]. Additionally, P450nor comprises the unique residues Ser286 and Asp393 that form the proton delivery pathway [26,27]. In contrast, the P450moxA possesses a highly hydrophobic substrate-binding pocket and possesses no such residues, suggesting a considerable functional divergence (Fig. 3). P450moxA can also be well superimposed on the 
substrate-free open form of the P450 PikC (CYP107L1 [29]) (PDB code, 2BJV chain B; $\mathrm{Z}$ score, 46.5; rmsd, $2.2 \AA$ for $378 \mathrm{C} \alpha$ atoms; sequence identity for fit regions, 39\%). The substrate-free (open) and substrate-bound (close) forms of the PikC structure were investigated, and it was revealed that binding of the bulky substrate narbomycin or YC-17 causes repositioning of the BC-loop and FG-helices [29]. Movement of the BC-loop and FG-helices is often observed in the P450 structure, and P450moxA might thus have a potential for such conformational changes when the substrate is bound. We also compared the structure of P450moxA with that of the human microsomal P450s CYP1A2 (PDB code, 2hi4), CYP2C8 (1pq2) [30], CYP3A4 (1tqn) [31] and CYP2D6 (2f9q) [32]. Although the overall structure of these P450s is primarily similar (Z score, $\sim 30.0)$, the low sequence identity $(\sim 18 \%)$ with ubiquitous sequence gaps results in a mutual conformational distortion of the P450 fold. Therefore, the substrate-binding sites of P450moxA and the microsomal P450s are not correctly superimposed. This indicates that the shared enzymatic properties of both types of P450s might have been acquired convergently.

\section{Substrate binding site}

The convincing electron density map enabled construction of the continuous model from the $\mathrm{N}$-terminus to the $\mathrm{C}$-terminus, and the structure of the substrate-binding pocket is well defined. The pocket above the heme is formed by the side-chains of Thr79, Leu80, Thr84, Leu88, Leu94, Leu96, Val295, Val396, and Ile397. The hydrophobic property of the substrate-binding pocket agrees with the substrate specificity of P450moxA, i.e., the P450moxA prefers highly hydrophobic compounds such as oleanolic acid and testosterone as substrates. In contrast, most of the hydrophobic residues of P450moxA are conserved neither in P450nor nor in the other known CYP105 P450s (Fig. 3). The crystal structures of P450nor reveal that the corresponding site comprises several hydrophilic side chains such as Arg64 and Arg174, which might play an important role in NADH recognition (Fig. 3).

As described above, P450moxA exhibits reasonable debenzylation activity with regard to L-BE. The reaction entails the simultaneous occupation of the benzyl group and luciferin moiety of L-BE adjacent to the heme iron, and thus, it requires a large space proximal to the heme. In the current structure, such a space is observed to be surrounded by the I-helix, Leu94, Leu96, Ala292, Val295, and Ile397. However, some 
of the P450s show a similar large space proximal to the heme, particularly in the substrate-free open form, despite lacking ability to act on bulky compounds. It is clear that, in order to understand the substrate recognition mechanism of P450s, the substrate complex structures need to be determined.

Unexpectedly, the current structure analysis reveals that the MES molecule occupies the substrate-binding site (Fig. 4). Model refinement indicates that although the average $B$-factor is high, MES is bound at the substrate-binding site with full occupancy. The side-chain hydroxyl group of Thr79 forms a hydrogen bond to the sulfate group of the MES molecule. Additionally, there are 2 water molecules (w505 and w520) near the sulfate group only in chain A, and these form a hydrogen bonding network to the side-chain amino group of Lys 70 and to the propionate group of the heme. The side-chains of Leu96 and Val295 and the heme are also present in close proximity to the MES molecule at a distance of 3.6-4.2 $\AA$, suggesting that van der Waals forces are responsible for these interactions. It is interesting to note that the morpholine ring $\mathrm{O} 1$ and $\mathrm{C} 4$ of the MES are located at a distance of approximately $4.5 \AA$ from the heme iron. No electron density peak was observed at the sixth coordination position of the heme iron, presumably due to the proximity between the morpholine ring and the heme iron. Therefore, the heme in the crystallized P450moxA largely exists in a five-coordinate high-spin state. The unexpected binding of the MES molecule might reflect the ability of $\mathrm{P} 450 \mathrm{mox} A$ with regard to capturing a broad range of structurally diverse compounds.

\section{Acknowledgements}

The authors would like to thank the beamline scientists at PF (Tsukuba, Japan) for their kind help during diffraction data collection. This work was supported by a research grant from the National Project on Protein Structural and Functional analyses from the Ministry of Education, Culture, Sports, Science, and Technology of Japan. 


\section{References}

[1] M.J. Coon, X.X. Ding, S.J. Pernecky, A.D. Vaz, Cytochrome P450: progress and predictions, FASEB J. 6 (1992) 669-673.

[2] P.R. Ortiz de Montellano, J.J. De Voss, Oxidizing species in the mechanism of cytochrome P450, Nat. Prod. Rep. 19 (2002) 477-493.

[3] A. Arisawa, A. Kumeda, World Patent WO2003/087381 (Oct. 23. 2003).

[4] H. Agematsu, N. Matsumoto, Y. Fujii, H. Kabumoto, S. Doi, K. Machida, J. Ishikawa, A. Arisawa, Hydroxylation of testosterone by bacterial cytochromes P450 using the Escherichia coli expression system, Biosci. Biotechnol. Biochem. 70 (2006) 307-311.

[5] T. Matsuoka, S. Miyakoshi, K. Tanzawa, K. Nakahara, M. Hosobuchi, N. Serizawa, Purification and characterization of cytochrome P-450sca from Streptomyces carbophilus, ML-236B (compactin) induces a cytochrome P-450sca in Streptomyces carbophilus that hydroxylates ML-236B to pravastatin sodium (CS-514), a tissue-selective inhibitor of 3-hydroxy-3-methylglutaryl-coenzyme-A reductase, Eur. J. Biochem. 184 (1989) 707-713.

[6] C.A. Omer, R. Lenstra, P.J. Litle, C. Dean, J.M. Tepperman, K.J. Leto, J.A. Romesser, D.P. O'Keefe, Genes for two herbicide-inducible cytochromes P-450 from Streptomyces griseolus, J. Bacteriol. 172 (1990) 3335-3345.

[7] N. Sawada, T. Sasaki, S. Yoneda, T. Kusudo, R. Shinkyo, M. Ohta, K. Inoue, Conversion of vitamin $\mathrm{D}_{3}$ to $1 \alpha, 25$-dihydroxyvitamin $\mathrm{D}_{3}$ by Streptomyces griseolus cytochrome P450SU-1, Biochem. Biophys. Res. Commun. 320 (2004) 156-164.

[8] F.S. Sariaslani, D.A. Kunz, Induction of cytochrome P-450 in Streptomyces griseus by soybean flour, Biochem. Biophys. Res. Commun. 141 (1986) 405-410.

[9] M. Taylor, D.C. Lamb, R. Cannell, M. Dawson, S.L. Kelly, Cytochrome P450105D1 (CYP105D1) from Streptomyces griseus: heterologous expression, activity, and activation effects of multiple xenobiotics, Biochem. Biophys. Res. Commun. 263 (1999) 838-842.

[10] Y. Fujii, S. Hirosue, T. Fujii, N. Matsumoto, H. Agematsu, A. Arisawa, Hydroxylation of oleanolic acid to queretaronic acid by cytochrome P450 from Nonomuraea recticatena, Biosci. Biotechnol. Biochem. 70 (2006) 2299-2302.

[11] D.F. Lewis, On the recognition of mammalian microsomal cytochrome P450 substrates and their characteristics: towards the prediction of human P450 substrate 
specificity and metabolism, Biochem Pharmacol. 60 (2000) 293-306.

[12] D.P. O’Keefe, P.A. Harder, Occurrence and biological function of cytochrome P450 monooxygenases in the actinomycetes, Mol. Microbiol. 5 (1991) 2099-2105.

[13] T. Nakashima, T. Tamura, A novel system for expressing recombinant proteins over a wide temperature range from 4 to $35^{\circ} \mathrm{C}$, Biotechnol. Bioeng. 86 (2004) 136-148.

[14] T. Nakashima, T. Tamura, Isolation and characterization of a rolling-circle-type plasmid from Rhodococcus erythropolis and application of the plasmid to multiple-recombinant-protein expression, Appl. Environ. Microbiol. 780 (2004) 5557-5568.

[15] I. Nagy, G. Schoofs, F. Compernolle, P. Proost, J. Vanderleyden, R. De Mot, Degradation of the thiocarbamate herbicide EPTC ( $S$-ethyl dipropylcarbamothioate) and biosafening by Rhodococcus sp. strain NI86/21 involve an inducible cytochrome P-450 system and aldehyde dehydrogenase, J. Bacteriol. 177 (1995) 676-687.

[16] A.G.W. Leslie, Recent changes to the MOSFLM package for processing film and image plate data, Joint CCP4/ESF-EACBM Newsl. Protein Crystallogr. 26 (1992).

[17] Collaborative Computational Project, The CCP4 suite: programs for protein crystallography, Acta Crystallogr. Sect. D Biol. Crystallogr. 50 (1994) 760-763.

[18] J. Navaza, AMoRe: an automated package for molecular replacement, J. Appl. Crystallogr. A 50 (1994) 157-163.

[19] J.R. Cupp-Vickery, C. Garcia, A. Hofacre, K. McGee-Estrada, Ketoconazole-induced conformational changes in the active site of cytochrome P450eryF, J. Mol. Biol. 311 (2001) 101-110.

[20] D.E. McRee, XtalView/Xfit—a versatile program for manipulating atomic coordinates and electron density, J. Struct. Biol. 125 (1999) 156-165.

[21] A.T. Brünger, P.D. Adams, G.M. Clore, W.L. DeLano, P. Gros, R.W. Grosse-Kunstleve, J.-S. Jiang, J. Kuszewski, M. Nilges, N.S. Pannu, R.J. Read, L.M. Rice, T. Simonson, G.L. Warren, Crystallography and NMR system (CNS): a new software suite for macromolecular structure determination, Acta crystallogr. Sect. D Biol. Crystallogr. 54 (1998) 905-921.

[22] J.J. Cali, D. Ma, M. Sobol, D.J. Simpson, S. Frackman, T.D. Good, W.J. Daily, D. Liu, Luminogenic cytochrome P450 assays, Expert Opin. Drug Metab. Toxicol. 2 (2006) 629-645. 
[23] F.P. Guegerich, Common and uncommon P450 reactions related to metabolism and chemical toxicity, Chem. Res. Toxicol. 14 (2001) 611-650.

[24] M.M. Harding, The architecture of metal coordination groups in proteins, Acta Crystallogr. Sect. D Biol. Crystallogr. 60 (2004) 849-859.

[25] L. Holm, C. Sander, Protein structure comparison by alignment of distance matrices, J. Mol. Biol. 233 (1993) 123-138.

[26] S-Y. Park, H. Shimizu, S. Adachi, A. Nakagawa, I. Tanaka, K. Nakahara, H. Shoun, E. Obayashi, H. Nakamura, T. Iizuka, Y. Shiro, Crystal structure of nitric oxide reductase from denitrifying fungus Fusarium oxysporum, Nat. Struct. Biol. 4 (1997) 827-832.

[27] H. Shimizu, E. Obayashi, Y. Gomi, H. Arakawa, S-Y. Park, H. Nakamura, S. Adachi, H. Shoun, Y. Shiro, Proton delivery in NO reduction by fungal nitric-oxide reductase: cryogenic crystallography, spectroscopy, and kinetics of ferric-NO complexes of wild-type and mutant enzymes, J. Biol. Chem. 275 (2000) 4816-4826.

[28] R. Oshima, S. Fushinobu, F. Su, L. Zhang, N. Takaya, H. Shoun, Structural evidence for direct hydride transfer from NADH to cytochrome P450nor, J. Mol. Biol. 342 (2004) 207-217.

[29] D.H. Sherman, S. Li, L.V. Yarmalitskaya, Y. Kim, J.A. Smith, M.R. Waterman, M.L. Podust, The structural basis for substrate anchoring, active site selectivity, and product formation by $\mathrm{P} 450 \mathrm{PikC}$ from Streptomyces venezuelae, J. Biol. Chem. 281 (2006) 26289-26297.

[30] G.A. Schoch, J.K. Yano, M.R. Wester, K.J. Griffin, C.D. Stout, E.F. Johnson, Structure of human microsomal cytochrome P450 2C8: evidence for a peripheral fatty acid binding site, J. Biol. Chem. 279 (2004) 9497-9503.

[31] J.K. Yano, M.R. Wester, G.A. Schoch, K.J. Griffin, C.D. Stout, E.F. Johnson, The structure of human microsomal cytochrome P450 3A4 determined by X-ray crystallography to 2.05-Å resolution, J. Biol. Chem. 279 (2004) 38091-38090.

[32] P. Rowland, F.E. Blaney, M.G. Smyth, J.J. Jones, V.R. Leydon, A.K. Oxbrow, C.J. Lewis, M.G. Tennant, S. Modi, D.S. Eggleston, R.J. Chenery, A.M. Bridges, Crystal structure of human cytochrome P450 2D6, J. Biol. Chem. 281, (2006) 7614-7622.

[33] J.D. Thompson, D.G. Higgins, T.J. Gibson, CLUSTAL W: improving the sensitivity 
of progressive multiple sequence alignment through sequence weighting, position-specific gap penalties and weight matrix choice, Nucleic Acids Res. 22 (1994) 4673-4680. 


\section{Figure legends:}

Figure 1. Expression of recombinant P450moxA and P450 assay using 6 luminogenic substrates. (A) SDS-PAGE analysis of cell-free extracts with and without thiostrepton induction. Arrows indicate P450moxA and TheD. CamB and TheC were not detected in this analysis due to their low molecular weight $(\sim 10 \mathrm{kDa})$. (B) Catalytic activity of P450moxA for 6 luminogenic substrates. The mean and standard errors for the quadruplicate assays are shown. RLU, relative light unit.

Figure 2. Structure of P450moxA. (A) Stereoview ribbon representation of the P450moxA structure. The model is colored according to the sequence by using a rainbow color ramp going from $\mathrm{N}$-terminus in blue to the C-terminus in red. Heme is shown as a sphere in dark red, and the bound MES molecule is represented as a stick model. Secondary structure elements have been labeled. (B) Stereoview superimposition among P450moxA (blue), P450nor (orange), and the P450 PikC substrate-free open form (green).

Figure 3. Multiple sequence alignment among the CYP105 and CYP55A1 P450s. Regions containing residues at the substrate-binding pocket are shown. Residues forming hydrophobic substrate-binding pocket of P450moxA highlighted in black are not conserved in the other P450s. Residues for NADH binding or for proton delivery in

the P450nor structure are highlighted in gray. Secondary structure elements of P450moxA have also been shown. The alignment was performed using CLUSTALW [33]. Identical, strongly similar, and weakly similar residues are denoted by asterisks, colons, and dots, respectively.

Figure 4. Stereoview of the substrate-binding site of P450moxA. Side-chains of the residues forming the substrate-binding site in the vicinity of the heme are represented by the stick model. Unbiased $m F_{\mathrm{O}}-D F_{\mathrm{c}}$ electron density map for the bound MES molecule is shown as a green mesh (contoured at $2.0 \sigma$ level). Water molecules near the sulfate moiety of MES (w505, w520, and w527) are also shown in red. The I-helix and $\mathrm{B}^{\prime}$-helix have been labeled. 


\section{Crystal structure of cytochrome P450 MoxA from Nonomuraea recticatena (CYP105)}

Yoshiaki Yasutake, Noriko Imoto, Yoshikazu Fujii, Tadashi Fujii, Akira Arisawa, and Tomohiro Tamura

Biochemical and Biophysical Research Communications, 2007

\section{Supplementary methods}

\section{Plasmid construction and expression in Rhodococcus erythropolis.}

Identification and cloning of the P450moxA gene (moxA) have been described previously [1]. The moxA gene and its downstream ferredoxin gene (mox $B)$ were inserted into the pTipQT vector [2,3] together with the $R$. erythropolis NI86/21 ferredoxin and ferredoxin reductase genes thc $C$ and $t h c D$ [4]. A ribosome-binding site (RBS) was added immediately upstream of $t h c C D$ in order to facilitate translation. The resulting plasmid pTipQT-MoxAB-ThcCD was introduced into the $R$. erythropolis JCM2301 cells by electropolation. The transformed cells were grown at $30^{\circ} \mathrm{C}$ in $100 \mathrm{ml}$ of Luria-Bertani (LB) medium containing $20 \mu \mathrm{g} / \mathrm{ml}$ tetracycline. The preculture was then inoculated into $1000 \mathrm{ml}$ of LB medium supplemented with $1 \mu \mathrm{g} / \mathrm{ml}$ thiostrepton for $24 \mathrm{hr}$ at $30^{\circ} \mathrm{C}$ to induce expression of MoxAB and ThcCD. The cells were harvested and resuspended in buffer containing $50 \mathrm{mM}$ Tris- $\mathrm{HCl}(\mathrm{pH} 7.4), 10 \%$ glycerol and 1 $\mathrm{mM}$ dithiothreitol (DTT). Cell lysis was performed using sonication, and the homogenate was clarified by centrifugation. Glycerol was added to the cell-free extracts to a final concentration of $20 \%$. The quantity of the expressed proteins was verified using sodium dodecyl sulfate-polyacrylamide gel electrophoresis (SDS-PAGE) and the Bradford protein assay (Bio-Rad).

Overexpression in E. coli and purification. For the X-ray structure analysis, a large amount of recombinant $\mathrm{P} 450$ moxA was produced using $E$. coli as the expression host. The gene encoding P450moxA (moxA) was amplified using polymerase chain reaction (PCR) with the following oligonucleotide primers incorporating the $N d e \mathrm{I}$ and SpeI sites (underlined) and C-terminal His-tag sequence: moxA-forward (NdeI) 5'-GCC

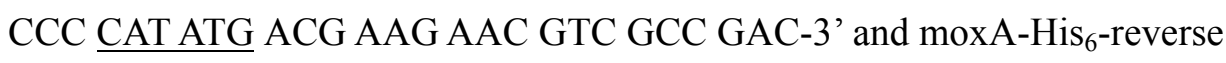




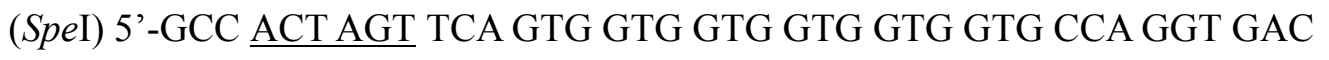
CGG GAG TTC GTG-3'. The amplified fragments were purified, digested with NdeI and SpeI, and cloned into the NdeI/SpeI sites of pT7NS-camAB [5]. The expression vector was transformed into the $E$. coli BL21 (DE3) strain. The cells were grown at $37^{\circ} \mathrm{C}$ for $3 \mathrm{hr}$, and ethanol and glycerol were then added to $3 \%$ and $0.8 \%$, respectively, to the culture medium for enhanced folding of P450moxA [6]. Overexpression was induced at $22^{\circ} \mathrm{C}$ for $22 \mathrm{hr}$ by the addition of $0.1 \mathrm{mM}$ isopropyl-1-thio- $\beta$-D-galactopyranoside (IPTG) to the culture medium supplemented with $80 \mu \mathrm{g} / \mathrm{ml} \delta$-aminolevulinic acid. The cells were harvested by centrifugation and resuspended in buffer A (50 mM Tris, pH 7.5, 10\% glycerol, 2 mM DTT). Lysozyme was added to a final concentration of $2 \mathrm{mg} / \mathrm{ml}$, and the suspension was maintained on ice for $30 \mathrm{~min}$. Subsequently, the cells were disrupted by sonication, and the insoluble debris was separated by centrifugation. The supernatant was dialyzed against Buffer B (50 mM Tris, pH 7.5, 10\% glycerol, $10 \mathrm{mM}$ imidazol). The sample was then applied to a Ni-NTA resin (QIAGEN), which had been pre-equilibrated with buffer B. The column was washed with buffer B, and the enzyme was eluted using a linear gradient of 10-400 $\mathrm{mM}$ imidazol in buffer B. The fractions were analyzed using SDS-PAGE. Peak fractions were pooled, dialyzed against a buffer containing $20 \mathrm{mM}$ Tris $\mathrm{pH} 7.5,10 \%$ glycerol and $50 \mathrm{mM} \mathrm{NaCl}$, and were concentrated to $20 \mathrm{mg} / \mathrm{ml}$, by using a centrifugal filtration device (Millipore). The CO difference spectral assay was performed to verify that the purified $\mathrm{P} 450 \mathrm{moxA}$ was in an active form [7]. The sample was stocked at $-80^{\circ} \mathrm{C}$ until use for crystallization.

Crystallization. Prior to crystallization, the glycerol was removed from the sample, and the protein concentration was adjusted to $8.0 \mathrm{mg} / \mathrm{ml}$ based on the results of the Pre-Crystallization Test (PCT; Hampton Research). The initial crystallization screening was performed by the sitting-drop vapor diffusion technique at $20^{\circ} \mathrm{C}$, using the commercially available sparse-matrix screens. Subsequent optimization of the initial conditions was performed by the hanging-drop vapor-diffusion at $20^{\circ} \mathrm{C}$ with varying the buffer $\mathrm{pH}$ conditions, precipitant concentrations, and the use of various additives. The rod-shaped single crystals suitable for an X-ray diffraction study $(0.3 \mathrm{~mm} \times 0.05 \mathrm{~mm} \times$ $0.05 \mathrm{~mm}$ ) were grown in hanging drops by mixing $1.8 \mu \mathrm{l}$ of the sample solution and 1.8 $\mu \mathrm{l}$ of the reservoir solution containing $0.1 \mathrm{M} 2$-( $N$-morpholino) ethanesulfonic acid 
(MES) (pH 6.5), 8\% ethyleneglycol, 3.5-6\% polyethylene glycol (PEG) 8000, and $15-20 \%$ glycerol.

Structure determination and refinement. The X-ray diffraction data were measured under cryogenic conditions $(100 \mathrm{~K})$ at beamline BL6A of the Photon Factory (PF; Tsukuba, Japan). All the diffraction spots were indexed and integrated with the program MOSFLM [8], and data scaling and merging were performed using the program SCALA [9]. The structure of P450moxA was solved by the molecular replacement (MR) method using the program AMoRe [10], applying the truncated structure of the P450eryF (CYP107A1; PDB code, 1jin) [11] as a search model. The MR solutions obtained revealed 2 molecules of P450moxA in the asymmetric unit (AU). The phase bias caused by the search model was reduced by density modification (solvent flattening, non-crystallographic symmetry (NCS) averaging, and histogram matching) by using the program DM [12]. The program XtalView/Xfit [13] was employed for model building and fitting. Atomic coordinates and isotropic $B$-factor refinement were performed using the program CNS ver. 1.1 [14] by applying the NCS restraint for the main-chain atoms (residues 15-74, 80-183, 197-339). The topology and parameter files for the bound ligands were obtained from the hic-up web site (http://xray.bmc.uu.se/hicup/) [15]. The stereochemical quality of the final refined model was verified by the program PROCHECK [16]. The data collection and refinement statistics are provided in Table 1. The atomic coordinates and structure factor amplitudes for P450moxA were deposited in the RCSB Protein Data Bank (http://www.rcsb.org) under accession code 2z36. Molecular illustrations (Figs 2 and 4) were prepared using the program PyMOL [17].

\section{References}

[1] H. Agematsu, N. Matsumoto, Y. Fujii, H. Kabumoto, S. Doi, K. Machida, J. Ishikawa, A. Arisawa, Hydroxylation of testosterone by bacterial cytochromes P450 using the Escherichia coli expression system, Biosci. Biotechnol. Biochem. 70 (2006) 307-311.

[2] T. Nakashima, T. Tamura, A novel system for expressing recombinant proteins over a wide temperature range from 4 to $35^{\circ} \mathrm{C}$, Biotechnol. Bioeng. 86 (2004) 136-148.

[3] T. Nakashima, T. Tamura, Isolation and characterization of a rolling-circle-type plasmid from Rhodococcus erythropolis and application of the plasmid to 
multiple-recombinant-protein expression, Appl. Environ. Microbiol. 780 (2004) 5557-5568.

[4] I. Nagy, G. Schoofs, F. Compernolle, P. Proost, J. Vanderleyden, R. De Mot, Degradation of the thiocarbamate herbicide EPTC ( $S$-ethyl dipropylcarbamothioate) and biosafening by Rhodococcus sp. strain NI86/21 involve an inducible cytochrome P-450 system and aldehyde dehydrogenase, J. Bacteriol. 177 (1995) 676-687.

[5] A. Arisawa, A. Kumeda, World Patent WO2003/087381 (Oct. 23. 2003).

[6] K. Kusano, M.R. Waterman, M. Sakaguchi, T. Omura, N. Kagawa, Protein synthesis inhibitors and ethanol selectively enhance heterologous expression of P450s and related proteins in Escherichia coli, Arch. Biochem. Biophys. 367 (1999) 129-136.

[7] T. Omura, R. Sato, The carbon monoxide-binding pigment of liver microsomes. I. Evidence for its hemoprotein nature, J. Biol. Chem. 239 (1964) 2370-2378.

[8] A.G.W. Leslie, Recent changes to the MOSFLM package for processing film and image plate data, Joint CCP4/ESF-EACBM Newsl. Protein Crystallogr. 26 (1992).

[9] Collaborative Computational Project, The CCP4 suite: programs for protein crystallography, Acta Crystallogr. Sect. D Biol. Crystallogr. 50 (1994) 760-763.

[10] J. Navaza, AMoRe: an automated package for molecular replacement, J. Appl. Crystallogr. A 50 (1994) 157-163.

[11] J.R. Cupp-Vickery, C. Garcia, A. Hofacre, K. McGee-Estrada, Ketoconazole-induced conformational changes in the active site of cytochrome P450eryF, J. Mol. Biol. 311 (2001) 101-110.

[12] K. Cowtan, DM: an automated procedure for phase improvement by density modification. Joint CCP4/ESF-EACBM Newsl. Protein Crystallogr. 31 (1994) 34-38.

[13] D.E. McRee, XtalView/Xfit - a versatile program for manipulating atomic coordinates and electron density, J. Struct. Biol. 125 (1999) 156-165.

[14] A.T. Brünger, P.D. Adams, G.M. Clore, W.L. DeLano, P. Gros, R.W. Grosse-Kunstleve, J.-S. Jiang, J. Kuszewski, M. Nilges, N.S. Pannu, R.J. Read, L.M. Rice, T. Simonson, G.L. Warren, Crystallography and NMR system (CNS): a new software suite for macromolecular structure determination, Acta crystallogr. Sect. D Biol. Crystallogr. 54 (1998) 905-921.

[15] G.J. Kleywegt, T.A. Jones, Databases in protein crystallography, Acta Crystallogr. 
Sect. D Biol. Crystallogr. 54 (1998) 1119-1131.

[16] R.A. Laskowsky, M.W. MacArthur, D.S. Moss, J.M. Thornton, PROCHECK: a program to check the stereochemical quality of protein structures, J. Appl. Crystallogr. 26 (1993) 283-291.

[17] W.L. Delano, The PyMOL molecular graphics system, DeLano Scientific, San Carlos, CA, USA (2002). 
(A)

(B)

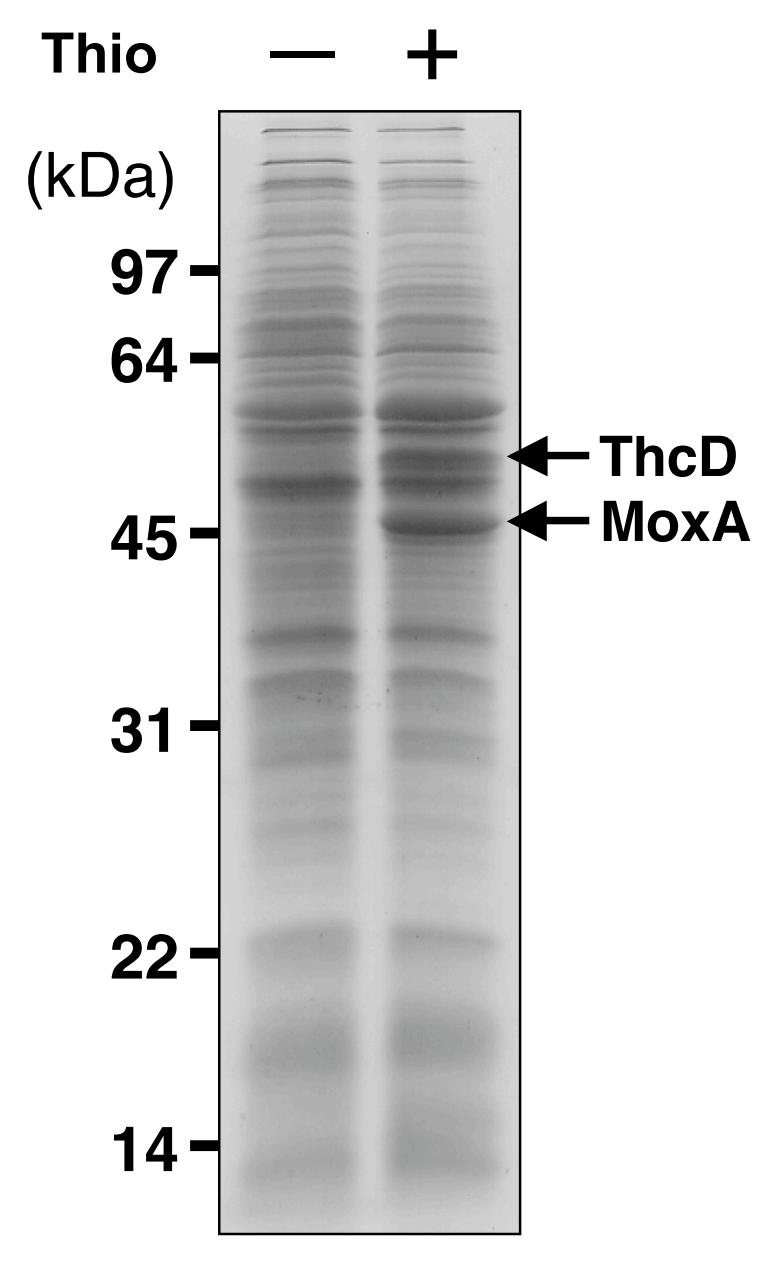

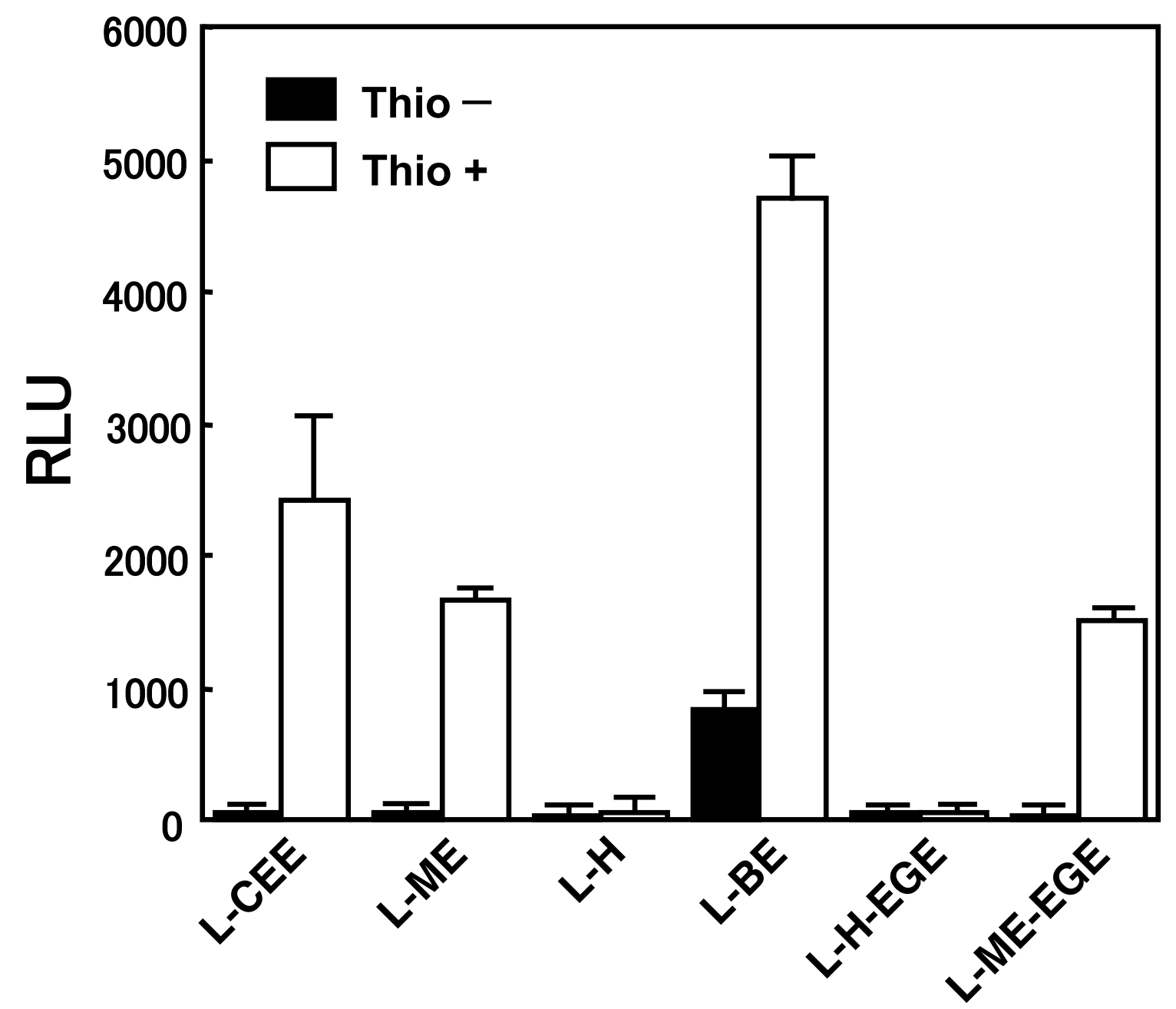

Substrate 
(A)
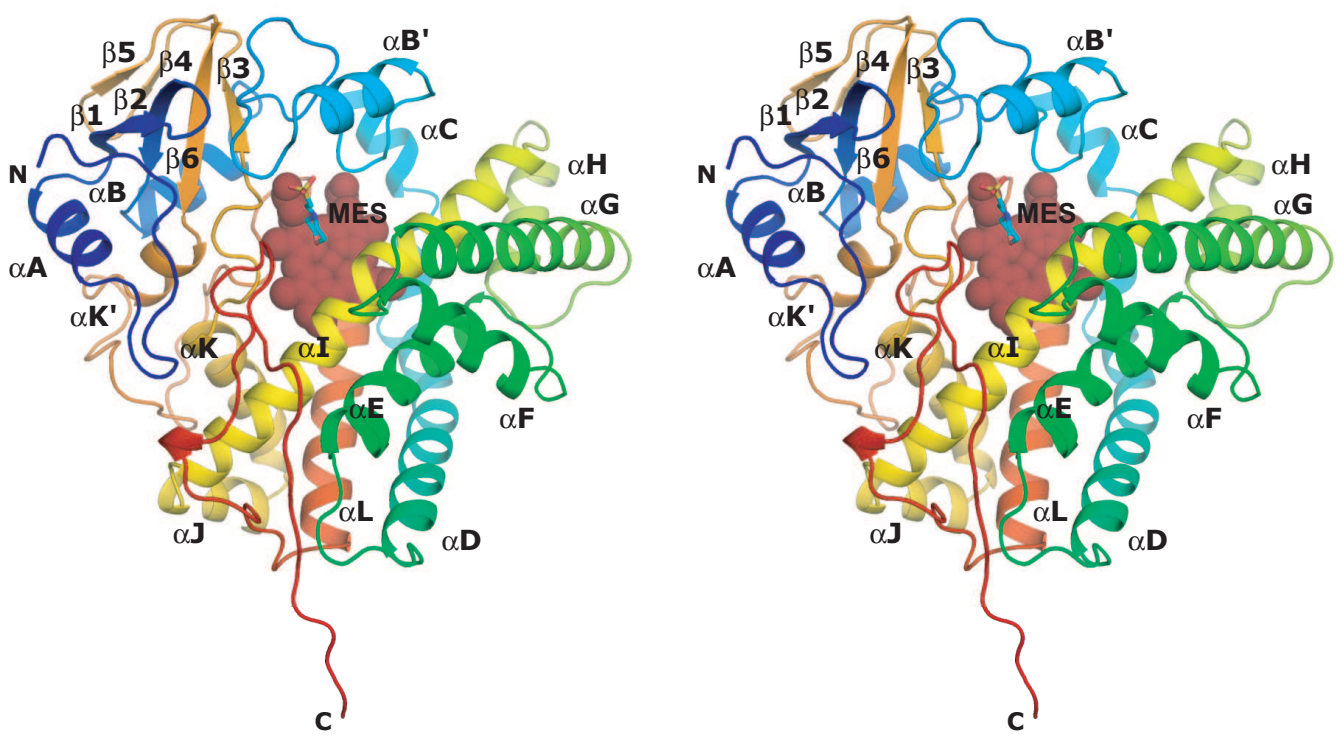

(B)

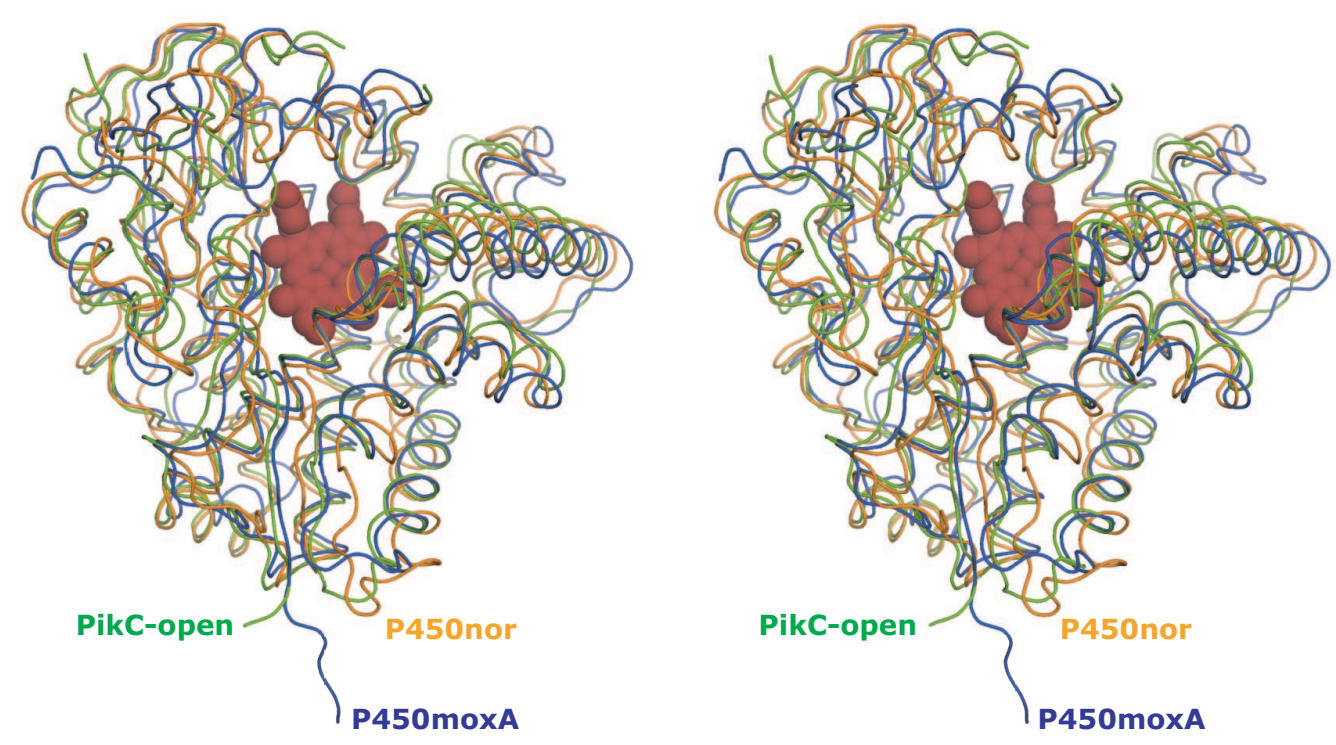




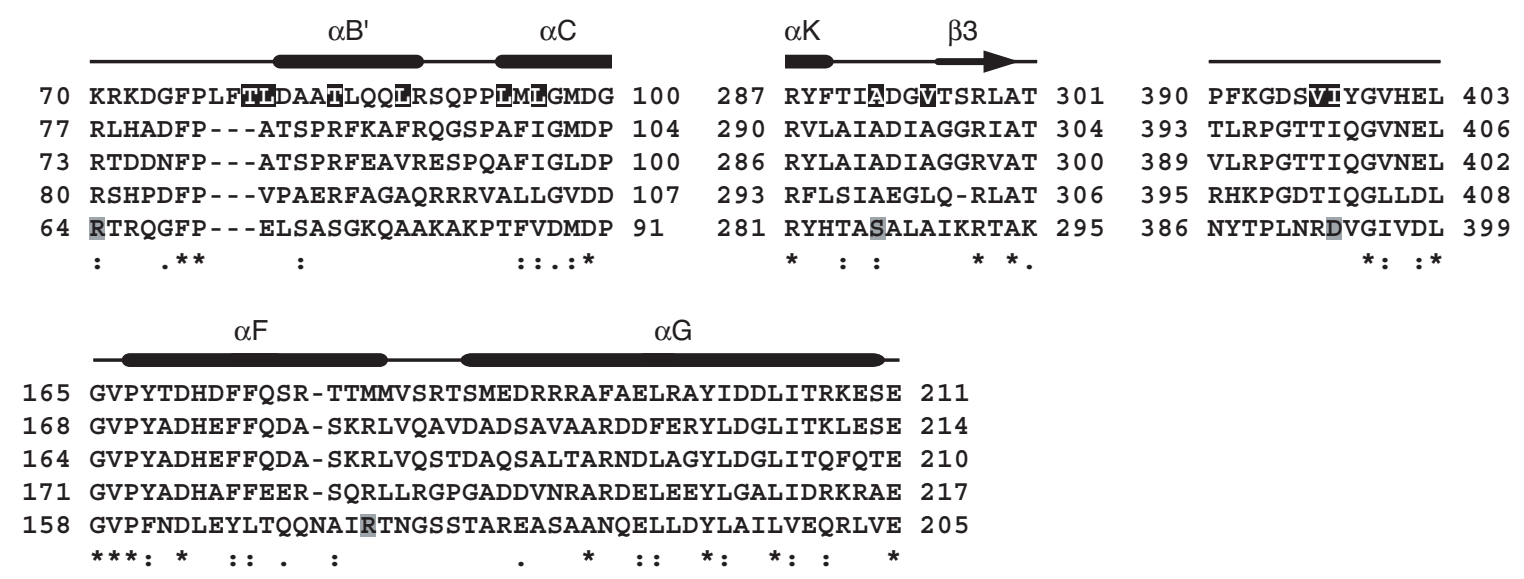




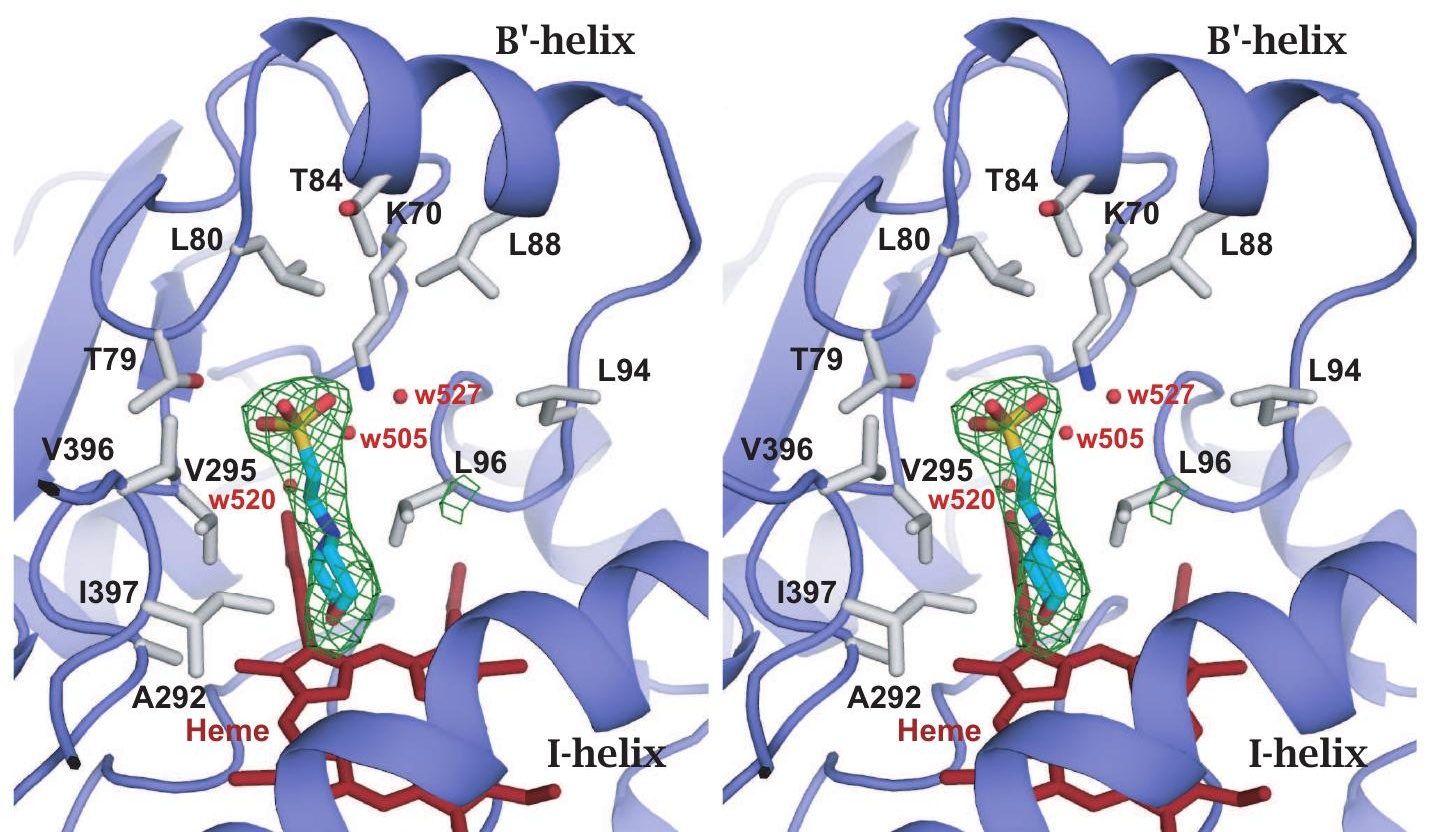


Table 1. Data-collection and refinement statistics.

\begin{tabular}{ll}
\hline Data collection statistics & \\
\hline Beamline & BL6A, PF \\
Wavelength $(\AA)$ & 0.97898 \\
Resolution $(\AA)$ & $50-2.8(2.95-2.80)^{\mathrm{a}}$ \\
Unit-cell dimensions & \\
$\quad a, b, c(\AA)$ & $81.6,83.3,175.9$ \\
$\quad \alpha, \beta, \gamma\left({ }^{\circ}\right)$ & $90.0,90.0,90.0$ \\
Space group & $P 22_{1} 2_{1}$ \\
Observed reflections & 147,143 \\
Unique reflections & 29,919 \\
$R_{\text {sym }}$ & $0.097(0.364)^{\mathrm{a}}$ \\
Completeness $(\%)$ & $99.3(100.0)^{\mathrm{a}}$ \\
Redundancy & $4.9(3.8)^{\mathrm{a}}$ \\
Mean $I / \sigma(I)$ & $7.0(2.0)^{\mathrm{a}}$ \\
\hline Refinement statistics and model quality & \\
\hline Resolution range $(\AA)$ & $50-2.8$ \\
$R_{\text {work }}{ }^{\mathrm{c}}$ & 0.230 \\
$R_{\text {free }}$ & 0.274 \\
Total number of atoms & 6,480 \\
Average $B$-factor $\left(\AA^{2}\right)$ & 48.8 \\
r.m.s.d. bond distances $(\AA)$ & 0.0058 \\
r.m.s.d. bond angles $\left({ }^{\circ}\right)$ & 1.25 \\
\hline & \\
\hline &
\end{tabular}

${ }^{a}$ Values in parentheses refer to data in the highest resolution shell (2.95-2.80 $\AA$ ).

${ }^{\mathrm{b}} R_{\mathrm{sym}}=\Sigma_{\mathrm{h}} \Sigma_{i}\left|I_{\mathrm{h}, i}-<I_{\mathrm{h}}>\right| / \Sigma_{\mathrm{h}} \Sigma_{i} I_{\mathrm{h}, i}$, where $<I_{\mathrm{h}}>$ is the mean intensity of a set of equivalent reflections.

${ }^{\mathrm{c}} R_{\text {work }}=\Sigma\left|F_{\text {obs }}-F_{\text {calc }}\right| / \Sigma F_{\text {obs }}$ for $95 \%$ of the reflection data used in the refinement. $F_{\text {obs }}$ and $F_{\text {calc }}$ are observed and calculated structure factor amplitudes, respectively.

${ }^{\mathrm{d}} R_{\text {free }}$ is the equivalent of $R_{\text {work }}$, except that it was calculated for a randomly chosen $5 \%$ test set excluded from refinement. 